\title{
Non-Model-Based Control of a Wheeled Vehicle Pulling Two Trailers to Provide Early Powered Mobility and Driving Experiences
}

\author{
David A. Sanders TD VR
}

\begin{abstract}
Non-model-based control of a wheeled vehicle pulling two trailers is proposed. It is a fun train for disabled children consisting of a locomotive and two carriages. The fun train has afforded opportunities for both disabled and able bodied young people to share an activity and has provided early driving experiences for disabled children; it has introduced them to assistive and powered mobility. The train is a nonlinear system and subject to nonholonomic kinematic constraints, so that position and state depend on the path taken to get there. The train is described, and then, a robust control algorithm using proportional-derivative filtered errors is proposed to control the locomotive. The controller was not dependent on an accurate model of the train, because the mass of the vehicle and two carriages changed depending on the number, size, and shape of children and wheelchair seats on the train. The controller was robust and stable in uncertainty. Results are presented to show the effectiveness of the approach, and the suggested control algorithm is shown to be acceptable without knowing the exact plant dynamics.
\end{abstract}

Index Terms-Powered mobility, nonholonomic constraint, wheelchair, control, driving, train.

\section{INTRODUCTION}

$\mathbf{T}$ HIS paper describes the design and control of a wheeled vehicle pulling two trailers. It is a fun train for disabled children consisting of a locomotive and two carriages that is providing early driving experiences for children with disabilities (Fig. 1.) and introducing powered mobility as an enjoyable and exciting experience [1].

Disabled people are keen to incorporate assistive technologies as they endeavor to improve their quality of life [1], [2], including using powered-mobility and wheelchairs [3], [4]. About 5,500,000 people in the USA have some form of paralysis [5] and some have tried to drive with the assistance of technology, but abandonment rates have been 35\%-75\% [6], [7].

The fun train has provided opportunities for able bodied and disabled children to participate and cooperate in something that involves sharing and taking turns. The control system described here means that new technology and mobility can be introduced with plenty of fun. Many disabled people cannot

Manuscript received March 23, 2017; revised June 25, 2017; accepted July 9, 2017. Date of publication July 12, 2017; date of current version January 8, 2018.

The author is with the University of Portsmouth, Portsmouth PO1 3DJ, U.K. (e-mail: david.sanders@port.ac.uk).

Digital Object Identifier 10.1109/TNSRE.2017.2726443

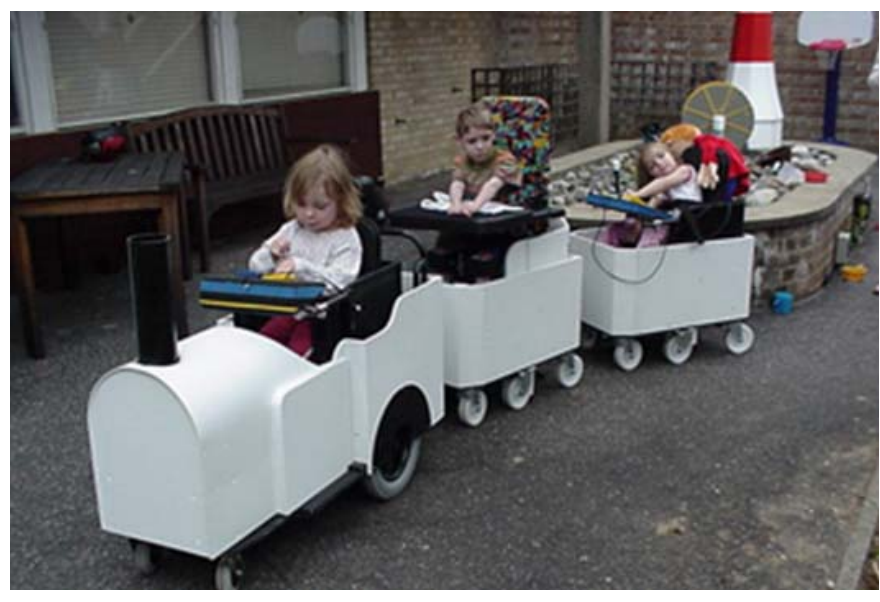

Fig. 1. Train locomotive towing two carriages.

handle a powered-wheelchair safely [1] but they can take part in activity on the locomotive and carriages.

An analysis of kinematic and dynamic models is in [8]. Wheeled vehicles are nonholonomic systems [9], [10] with kinematic constraints that cannot be integrated and therefore cannot be removed from equations. Tools for exploring and controlling nonholonomic systems are presented in [11]. Using Lagrange and differential geometry, a general dynamical model can be derived for vehicles with nonholonomic constraints [11]-[13]. Many controllers and models have been described but they have usually integrated kinematic and dynamic controllers or used kinematic trajectory tracking to follow desired trajectories.

Work in [14] and [15] discussed controlling nonholonomic mobile robots using path tracking methods and models of their kinematics in 2-D polar coordinates. A predictive control algorithm that used a model of the target system is described in [16]-[18] describe analysis of a mobile robot towing two carriages (including nonholonomic constraintsbased feedback).

Control of wheeled robots and wheelchairs [8]-[10], [13], [14], [16]-[19] have been widely investigated, but little research has considered dynamic control. Torque control has often been considered and research about model-based control has been described, such as adaptive dynamic control of nonholonomic mobile robots [20]. Martins et al. [21] describe 
an adaptive controller for a unicycle robot. Adaptive neural sliding mode control for trajectory tracking of wheeled vehicles is presented in [22] and tracking control for a vehicle is described in [23]. Robust adaptive feedback linearizing dynamic controller is proposed in [24].

Model-based control needs an accurate model of the target system and that can be difficult to obtain. Algorithms that do not use an arithmetic model need fewer computer operations. Because it may be difficult to obtain an accurate mathematical model, it is often better to design controllers that do not depend on the target system dynamics [25]. There has been research about non-model based control of wheeled vehicles. In [26] an algorithm for a fuzzy controller was used to control vehicles with two independent wheels. ANN control is described in [27] for a mobile robot and [28] describes a controller based on a modified transpose Jacobian method for tracking a locomotivecarriage type vehicle (without having a model of the target system).

A controller that is not based on having an accurate model of the train is described here as the train characteristics change as different people and numbers of people ride in it. The controller compares well with model-based algorithms but that is independent of the dynamic models of the locomotive towing two carriages - Fig. 1. The controller needs less computer power and is robust to uncertainty.

This paper introduces control for a nonholonomic wheeled locomotive and two carriages. Barbalat's lemma has been used with Lyapunov to confirm the stability of the closedloop controller and system. That analysis is available from the author on request. Finally, results from some simulated and real time experiments are presented. Results show that the proposed method is adequate and suitable for the vehicle and two carriages.

Important achievements are:

- Design of the fun train (locomotive and two carriages).

- Dynamics modelling of the train.

- Non-model-based dynamic control.

- Testing the control to check reduction in tracking error.

\section{The Vehicle and Two Carriages}

A train is an icon of freedom of movement and is loved by children. A driver conventionally has responsibility for transporting passengers and therefore has a sense of obligation and authority. Children could feel that they were a 'train driver' in the locomotive and safely drive other kids around as passengers.

The fun train was designed to be used by as many children with special needs as possible. It needed to accommodate most wheelchair seating systems. Three seat adaptors were needed to house all the various seating arrangements. Figure 2 shows a carriage with the adjustable seat shell and without the cover for the front.

A standard child CAPPs seat is mounted on the seat shell. The CAPPS is fitted within the seat shells in the locomotive and carriages as well as an in house adaptable cushioned seat for nursery school kids.

If the locomotive structure had been removable then it might have been heavy or could have been lost, so the locomotive

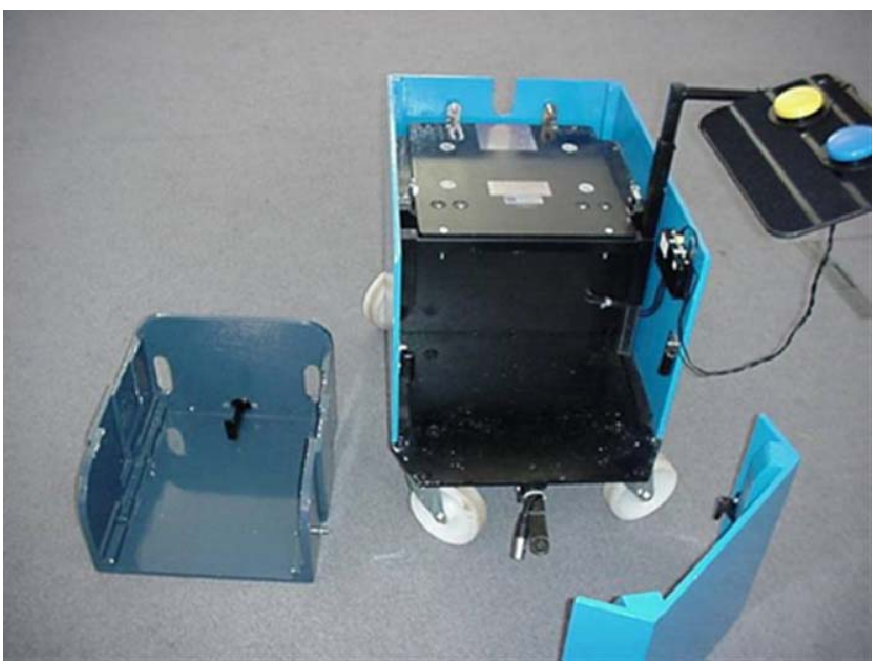

Fig. 2. Open access to the carriages.

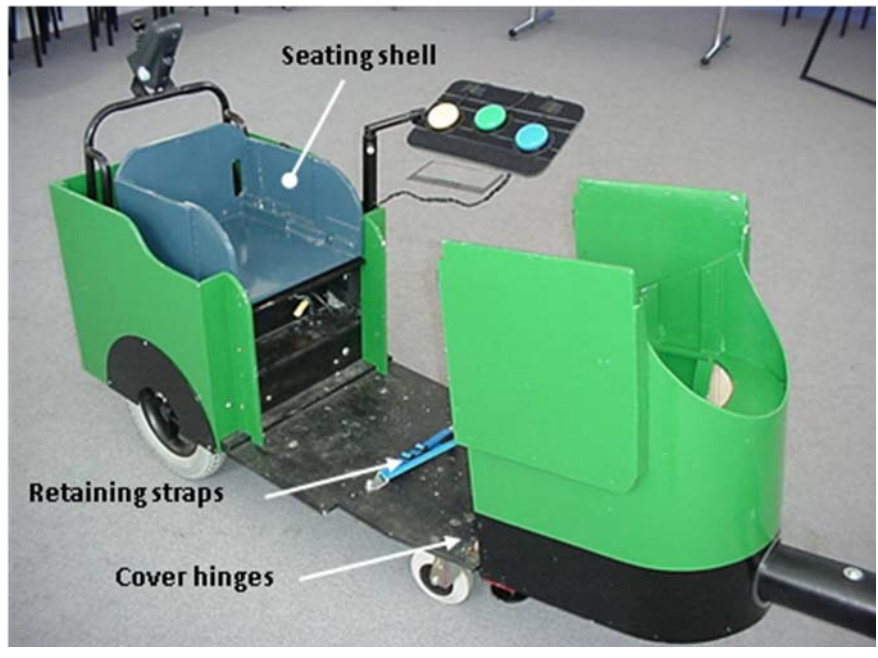

Fig. 3. Locomotive in open access.

was made of wood and connected by a hinge at the front. The hinge provided access to the seating area. The main body was fixed and could not be removed as the bodywork was lighter and the locomotive could have tipped without it as weight was centered above the rear driving wheels for traction and grip.

A purpose made sub frame and chassis was designed and built to create a locomotive. A standard controller, batteries and wheelchair motors were used (supplied directly from a wheelchair manufacture).

The locomotive is shown open in Fig. 3.

A 'Newton Badger Vixen' wheelchair chassis was used as a framework for the imitation steam engine. The wheelbase dimensions were changed to consider:

- Effects due to changing multiple loads with various numbers and sizes of passengers in the carriages.

- Rolling on steeper slopes.

- Drive tracking.

- Distribution of weight.

- Proportions of width to length to produce lifelike and convincing locomotive looks. 


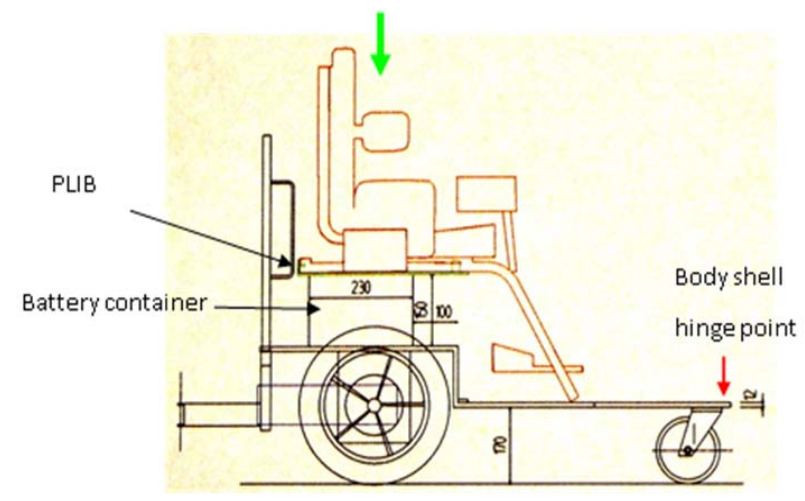

Fig. 4. Locomotive sub-assembly.

Tests were conducted to check clearances, especially in a new adventure tunnel that had some smaller gaps and restricted spaces. The locomotive needed to look reasonably realistic and that defined shape and then size (keeping the length to width ratio) while allowing children to safely ride along corridors. Figure 4 shows an illustration of the locomotive. The thick down arrow at the top shows the focus of the weight above and down on to the driving wheels to give purchase, especially if pulling children in the carriages.

A box was placed under the PLIB to hold the batteries. The outer covering isn't shown in Fig. 4. However the smaller down arrow at the front points to the positions of the hinges. Hinging the bodywork provided a counter weight for the front casters. If the bodywork had been completely removable, the lack of any weight above the casters at the front could have caused some instability.

When the bodywork was down and locked in the working position, space was still available for any trays that might be required. Two choices were provided for the trays: a molded tray for a CAPPS seat with tray fixing points within the seating system and tray mounting supports for integrated switch trays.

The train is to be equipped with a Langner Scanning Collision Avoidance Device (langner-SCAD) [1] at the front of the train locomotive to detect local obstacles and stop the train. For further reading, obstacle avoidance for powered wheelchairs is described in [29]-[32].

From the outset, the train has offered new opportunities for children with complex needs: The locomotive can be driven by a child driver with only a single switch to start and stop the train. Velcro allows switch positions to be quickly and easily adjusted to suit different children and it was a safe and soft fixative. A platform is also used to more permanently mount the switches. It incorporated adjustable clamps and bolts within slots to secure the input devices so that bespoke arrangements could be created for specific users.

When testing began with the prototypes of the train, all of the children who saw or heard it were interested. Because both disabled and non-disabled kids were interested, the ability to accommodate all sorts of different seating arrangements was included in a redesign and children from both groups could operate or ride in the train. Almost all the children at Chailey Heritage School had a Chailey Adjustable Postural Support Seating System (CAPSS) to provide the specific postural support required by each child.
The carriages and the locomotive had mounting assemblies built in for general and specialist seating and chairs.

The adaptability of the train seating allowed kids with varying ability to be a passenger. Some children wanted to drive the train and others wanted to be a passenger. A few children just wanted to watch, although that was often because all the carriages were full. Only three children could ride in the train at any one time but some children would follow the train in their powered wheelchairs and staff pushed children behind the train while they were sitting in their wheelchairs or buggies.

The use of the train was an extended group activity. The movement and overall control of the whole train affected all the passengers and the children following the train. Some children obviously grasped what was going on, especially the children who could already drive a powered wheelchair. Children who were not disabled could also ride in the train with young disabled or non-disabled passengers.

Features were separated and each kiddy riding on the vehicle and two carriages could perform a range of tasks. Driving using a go / stop switch was the main control function but children on the train could control devices around the vehicle and two carriages. Carriages had infra-red transmitters that could interact with toys and responsive systems in play areas and adventurous tunnels. An infrared beam would be perceived and identified by a receiver within a steel conduit mounted close to the floor.

\section{Control Strategy}

Controls could be divided up and that provided children with the option of controlling different things on and from the toad train. The train driver was responsible for starting and stopping. Other passengers could be given control of environmental gadgets and contraptions that the train might encounter (bubble blowers, merry go rounds, waterjets etc). The train also had a whistle and one of the passengers could control that. The locomotive also had a noise maker that provided the simulated sound of a steam locomotive.

Switches were connected directly to make things straightforward and to make the electrical connections simpler. Loading and unloading children could be time consuming so the switches needed to be "plug-and-play" to reduce waiting time as the children were usually excited and could be impatient.

Control could be reduced if necessary and for example, the infra-red switch could be overridden so that it was always switched on and activated any infra-red devices the train went past, or the train could be restricted to following a wire in the floor. In those cases, children would not have to worry about operating manual switches and that could stimulate children who were unaware or did not understand switches.

A difficulty with offering numerous control options was the need for clinical assistants and helpers to connect up and disconnect switches and so the arrangement had to be intuitively obvious and easy to do. Figure 5 shows the system and how the auxiliary functions could be shared between the driver and the passengers (between the locomotive and the carriages). Each carriage had an in-line to provide a coupling point. The infrared communications delivered more autonomy 


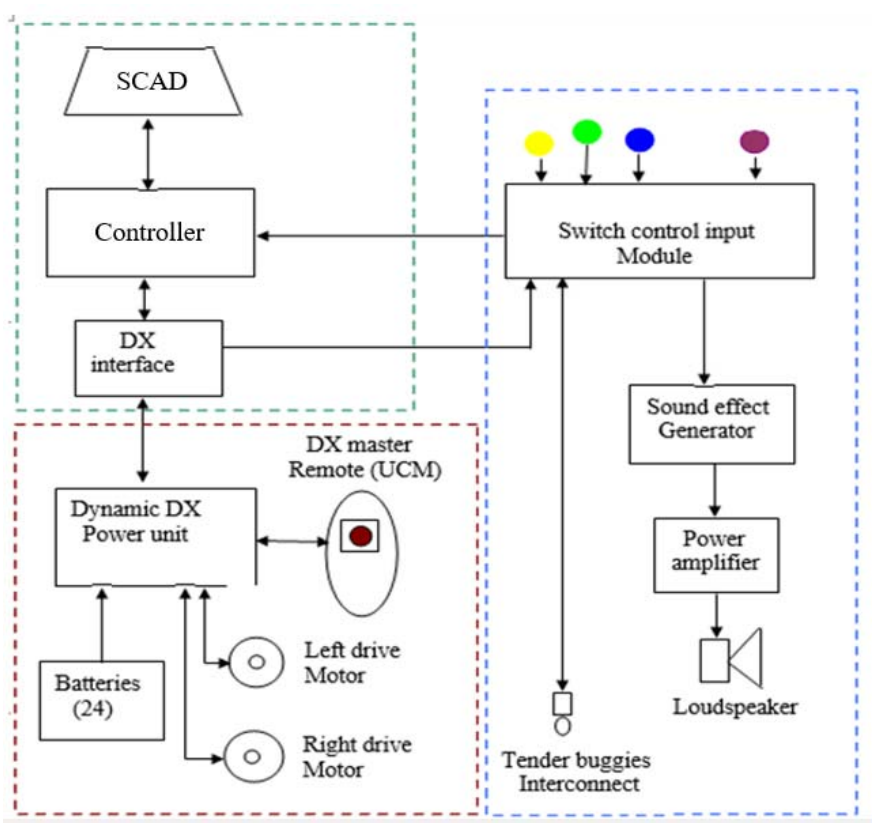

Fig. 5. Control distribution between the passengers for off track movement.

and expanded choices and the number of devices that could be operated.

In Fig. 5, the outlined area on the right shows how switch control was distributed via an inter-connection interface so that each child on the train could control something.

A sound effect generator was also included. It was connected to the drive so that the locomotive made steam engine noises. Single jack sockets provided control options and switches could be plugged in and directly connected. The locomotive had all the possible connection options as the locomotive could be used as a standalone device, as well as with one or two carriages attached.

The outlined area on the bottom left in Fig. 5 encloses the power amplifiers for the motors that converted the torque demand from the controller to current into the two drive motors. The outlined area at the top left in Fig. 5 encloses the controller proposed in this paper and the Langner-SCAD.

The train has been restricted to a track route originally designed for wheelchairs. Further developments have allowed some wheelchair users to safely move away from the track. A controller is described in the next Section that will allow the train to safely move away from the track.

\section{Controller}

The wheeled locomotive and the two carriages consist of a module at the front with differential drive for the driving wheels pulling two simple carriages. The locomotive has two separately actuated driving wheels at the rear and two extra small wheels at the front to support the weight of the locomotive.

Using nomenclature based on [22], the locomotive and the middle carriage are connected at point $P$, a central point between the driving wheels of the locomotive. The middle

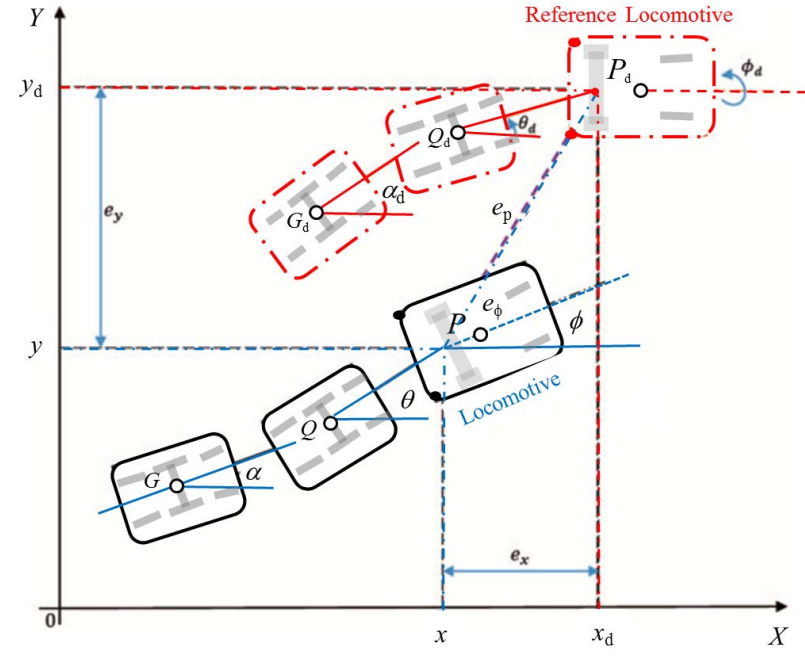

Fig. 6. Simulated vehicle and two carriages and simulated trajectory for the vehicle and two carriages.

carriage and the rear carriage are connected at point $Q$. Angular displacement of the locomotive driving wheels is denoted by $\theta_{\text {right }}$ and $\theta_{\text {left }}$. The radius of the locomotive wheels is $r_{\text {loco. }}$. If point $P$ is the center of the locomotive then coordinates of point $P$ can be represented by $\left(x_{l o c}, y_{l o c}\right)$. The orientation of the locomotive is $\phi$, the orientation of the middle carriage is $\theta$ and the orientation of the rear carriage is $\alpha . L_{\text {loco }}$ is the length from point $P$ to the locomotive center of gravity and $L_{\text {middle }}$ is the length from point $Q$ to the center of gravity of the centre carriage. $L_{\text {rear }}$ is the length from point $G$ to the center of gravity of the rear carriage.

The gap between the locomotive driving wheels is $2 h$, the distance between the middle carriage wheels is $2 h_{1}$ and the distance between the middle carriage wheels is $2 h_{2} . d$ is the length from the middle carriage wheels to point $P$ and $b$ is the length from the middle carriage wheels to the point $Q$. The masses of the locomotive, middle carriage and rear carriage were $\mathrm{m} 1, \mathrm{~m} 2$, and $\mathrm{m} 3 . J_{1}, J_{2}$, and $J_{3}$ are the moments of inertia of the locomotive, middle carriage and rearmost carriage. So, the position and pose of the vehicle and two carriages can be expressed as:

$$
\boldsymbol{q}=\left[x_{l o c}, y_{l o c}, \phi, \theta, \alpha .\right]^{\mathrm{T}} \text {. }
$$

The dynamic modelling of the vehicle and two carriages is portrayed in Appendix A. The control algorithm uses PD-action filtered errors for the vehicle and two carriages with a locomotive and two carriages. A representation of the locomotive and carriages is shown in in Fig. 17 in Appendix A. Figure 6 shows the position error $\left(e_{p}\right)$ and orientation error of the locomotive $\left(\mathrm{e}_{\phi}\right)$.

The position error $\left(\mathrm{e}_{\mathrm{p}}\right)$ and orientation error of the locomotive $\left(\mathrm{e}_{\phi}\right)$ are defined as:

$$
\begin{aligned}
& e_{\mathrm{p}}=\sqrt{ }\left(e_{\mathrm{x}}^{2}+e_{\mathrm{y}}^{2}\right) \\
& e_{\phi}=\tan ^{-1}\left(e_{y} / e_{\mathrm{x}}\right)-\phi
\end{aligned}
$$




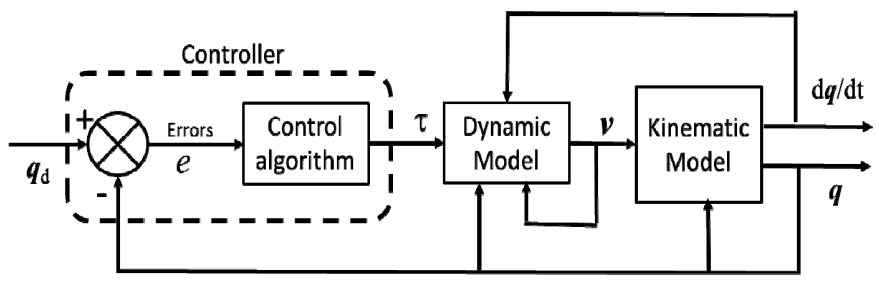

Fig. 7. The closed-loop controller for testing.

Where

$$
\begin{array}{r}
e_{\mathrm{x}}=x_{\mathrm{d}}-x=e_{\mathrm{p}} \cos \left(\phi+e_{\phi}\right) \\
\text { ey }=y_{\mathrm{d}}-y=e_{\mathrm{p}} \sin \left(\phi+e_{\phi}\right)
\end{array}
$$

Locomotive position error $\left(e_{\mathrm{p}}\right)$ and locomotive orientation error $\left(e_{\phi}\right)$ should tend to zero and then remain there.

The locomotive and carriages were tested with simulated trajectories and by driving with joysticks and switches. The closed-loop controller configuration used is shown in Fig. 7.

The right and left actuator torques for the motors were:

If $|e \phi|<\delta$ then:

$$
\begin{aligned}
\tau_{\text {right }} & =\mathrm{K} 1_{\mathrm{pos}} e_{\mathrm{pos}}+\mathrm{K} 1_{\mathrm{sp}}\left(\mathrm{d} e_{\mathrm{pos}} / \mathrm{dt}\right)+\mathrm{K} 2_{\mathrm{pos}} e_{\phi}+\mathrm{K} 2_{\mathrm{sp}}\left(\mathrm{d} e_{\phi} / \mathrm{dt}\right) \\
\tau_{\text {left }} & =\mathrm{K} 1_{\mathrm{pos}} e_{\mathrm{pos}}+\mathrm{K} 1_{\mathrm{sp}}\left(\mathrm{d} e_{\mathrm{pos}} / \mathrm{dt}\right)-\mathrm{K} 2_{\mathrm{pos}} e_{\phi}-\mathrm{K} 2_{\mathrm{sp}}\left(\mathrm{d} e_{\phi} / \mathrm{dt}\right)
\end{aligned}
$$

If $|e \phi|>=\delta$ then:

$$
\begin{array}{r}
\tau_{\text {right }}=\mathrm{K} 2_{\text {pos }} e_{\phi}+\mathrm{K} 2_{\mathrm{sp}}\left(\mathrm{d} e_{\phi} / \mathrm{dt}\right) \\
\tau_{\text {left }}=-\mathrm{K} 2_{\mathrm{pos}} e_{\phi}-\mathrm{K} 2_{\mathrm{sp}}\left(\mathrm{d} e_{\phi} / \mathrm{dt}\right)
\end{array}
$$

Where, $\mathrm{Kn}_{\mathrm{pos}}$ and $\mathrm{Kn}_{\mathrm{sp}}$ are proportional and speed gains for the controller and $\delta$ is a small preset value.

IF direction error, $|e \phi|$, is above a preset value, $\delta$, then following equations (7) and (8), only direction error will be considered and the controller will turn the locomotive to reduce $|e \phi|$.

ELSE IF orientation error, $|e \phi|$, is below the present value, $\delta$, then following equations (5) and (6), position and direction will both be controlled.

\section{TEsting And Results}

The configuration of the vehicle and two carriages was expressed as a generalized coordinate vector

$$
\boldsymbol{q}=[x, y, \phi, \theta, \alpha \cdot]^{\mathrm{T}} .
$$

The initial conditions were

$$
\boldsymbol{q}_{0}=\left[\begin{array}{lllll}
0 & 0 & 3 \pi / 2 & 0 & 0
\end{array}\right]
$$

A circular reference path for the second carriage was used as an input to a simulation in Matlab / Simulink.

$$
z_{\mathrm{d}}(\mathrm{t})=0.8 \cos \mathrm{t}, \quad y_{\mathrm{d}}(\mathrm{t})=0.8 \sin (\mathrm{t})
$$

Controller gains for the proposed controller were set as

$$
K 1_{\text {pos }}=1, \quad K 1_{\text {sp }}=1, \quad K 2_{\text {pos }}=0: 5, \quad K 2_{\text {sp }}=0.5
$$

The circular reference path is shown in Fig. 8.

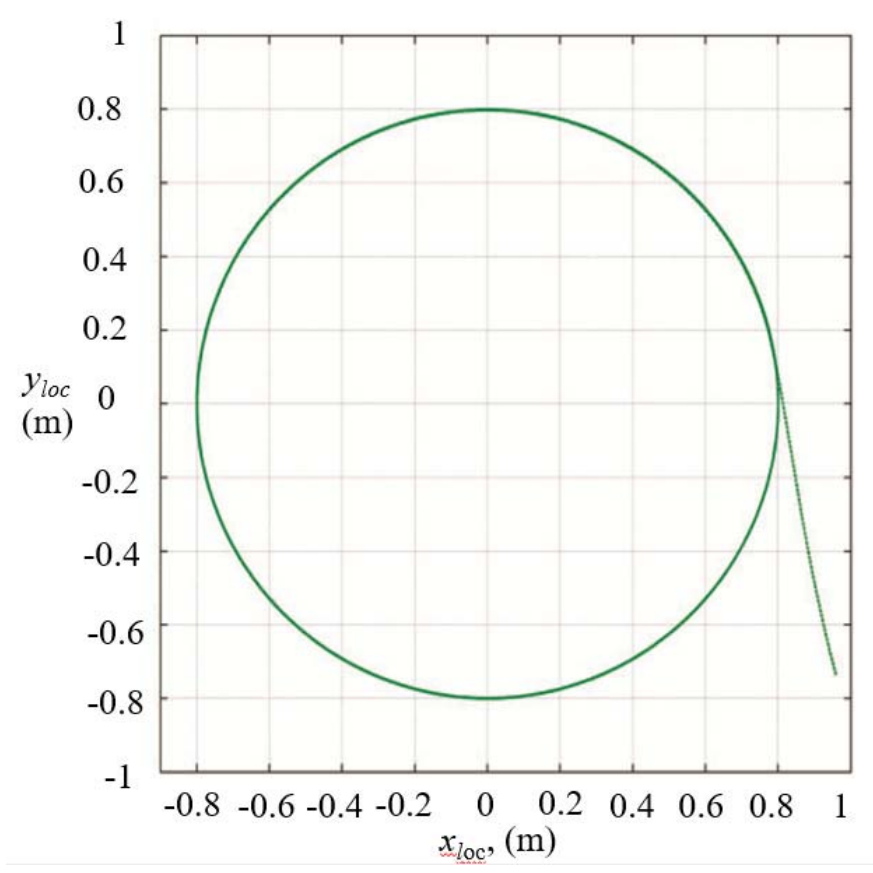

Fig. 8. The circular reference path.
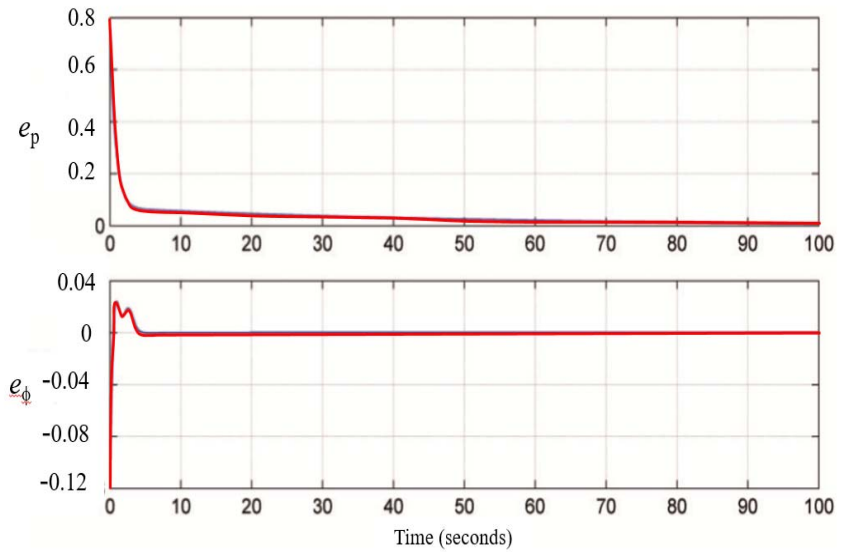

Fig. 9. Errors in tracking: Graph above is position error (epos $(\mathrm{m}))$; Graph below is orientation error $\left(e_{\phi}(\mathrm{rad})\right)$.
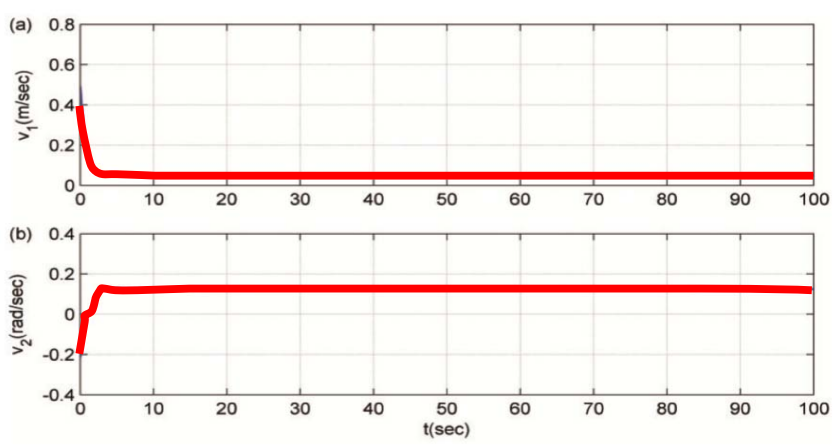

Fig. 10. Kinematic control inputs: (a) v1 (m/s); (b) v2 (rad/s).

The tracking was simulated and the resultant tracking errors are shown in Fig. 9.

Kinematic control inputs are shown in Figure 10.

A fixed camera with processing rate of 30 frames per second and resolution of $480 \times 640$ pixels was mounted near the ceiling and connected to a computer. DC servo motors were 


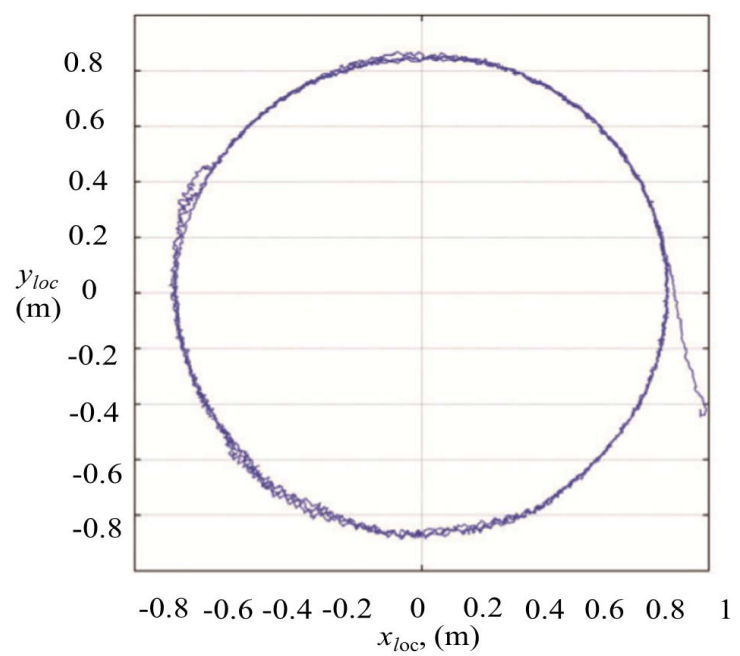

Fig. 11. Tractor reference point path.

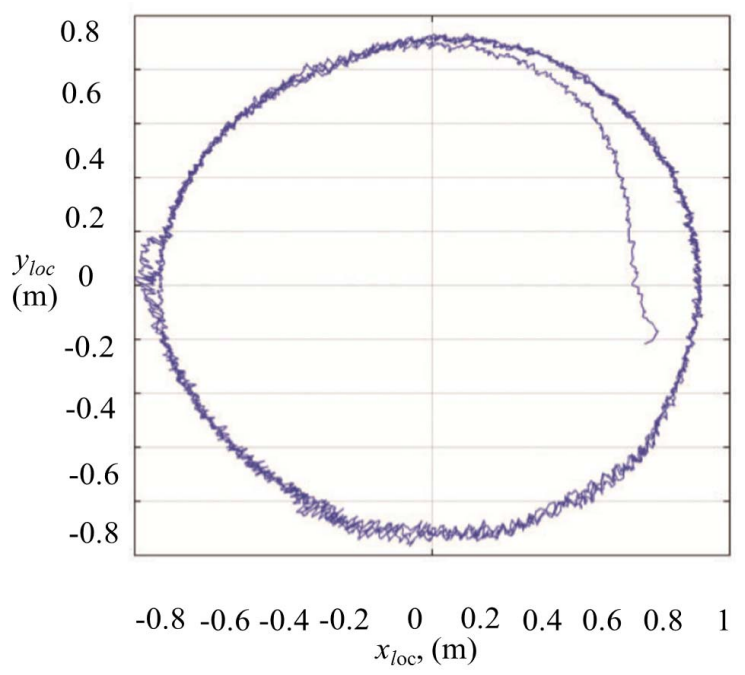

Fig. 12. Middle carriage reference point path.

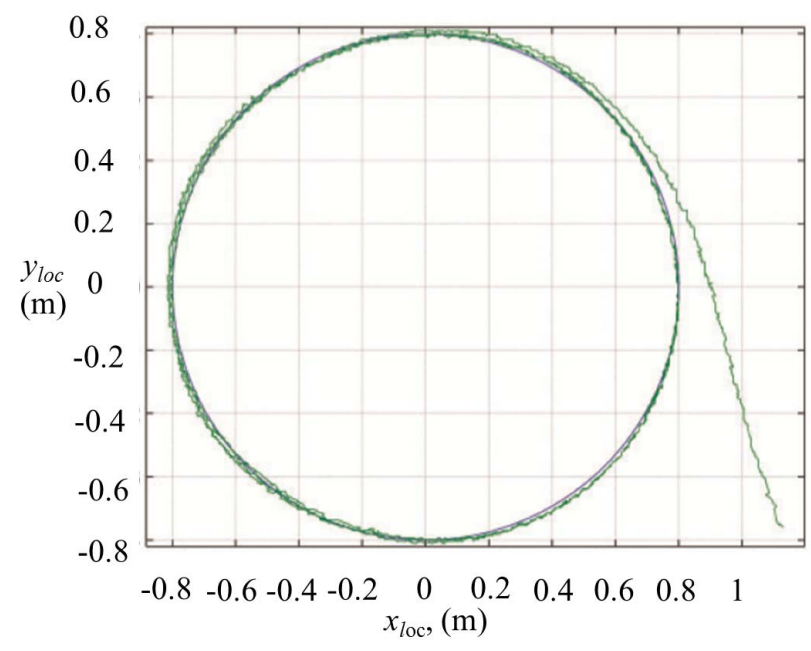

Fig. 13. Second carriage reference point path.

the actuators and they were connected directly to the wheels of the locomotive.

The train was made to follow reference trajectories in order to validate the proposed controller. The trajectories were
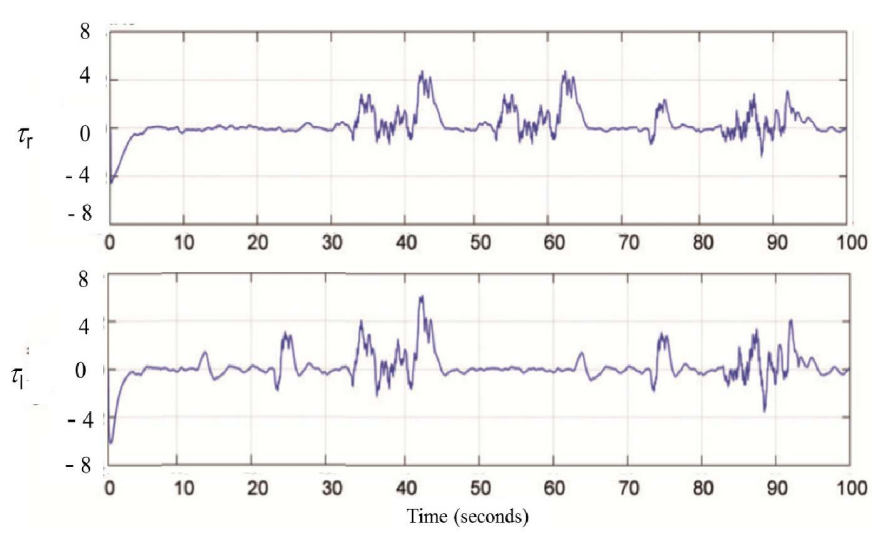

Fig. 14. Actuator torques: (top) right wheel ( $\mathrm{N} / \mathrm{m})$; (bottom) left wheel $(\mathrm{N} / \mathrm{m})$.
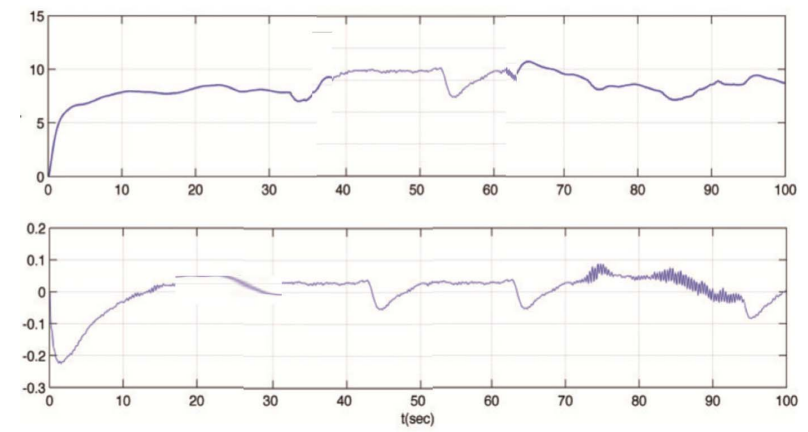

Fig. 15. Tracking errors for second carriage: At the top is $e_{x}(m)$, and at the bottom is $e_{y}(m)$.
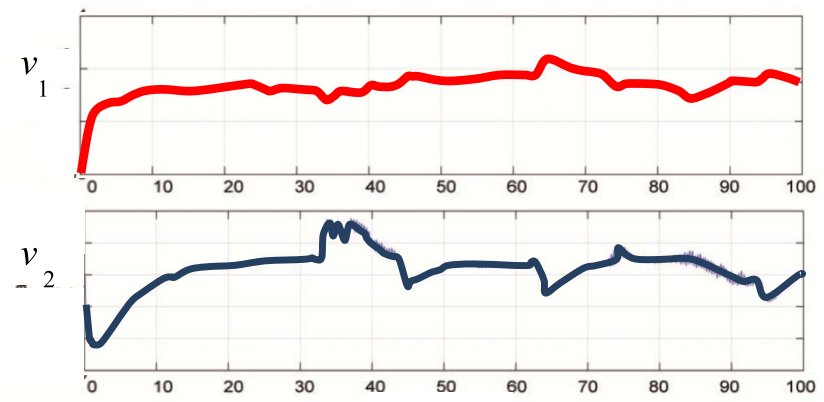

Fig. 16. Kinematic control inputs: At the top in red is $\mathrm{v}_{1}(\mathrm{~m} / \mathrm{s})$ and below in blue is $v_{2}(\mathrm{rad} / \mathrm{s})$.

generated in Cartesian coordinates by a planner. The train started from $\boldsymbol{q}_{0}$ and followed circular reference trajectories.

A vision system estimated posture and transmitted that to the computer. Sampling time was limited by the vision system processing speed. Sampling time (and processing speed) was $33 \mathrm{~ms}$. Control commands were generated by the computer and sent to the locomotive through a USB cable. Control software was developed in MATLAB \Simulink.

The locomotive and two carriages started from q0 (9) and followed a circular reference trajectory (10). Paths for the locomotive, middle carriage and second carriage, are shown in Figs 11 to 13.

Figure 14 shows the right and left actuator torques and Fig. 15 shows the kinematic control inputs.

Tracking errors for the second carriage are shown in Fig 16. 


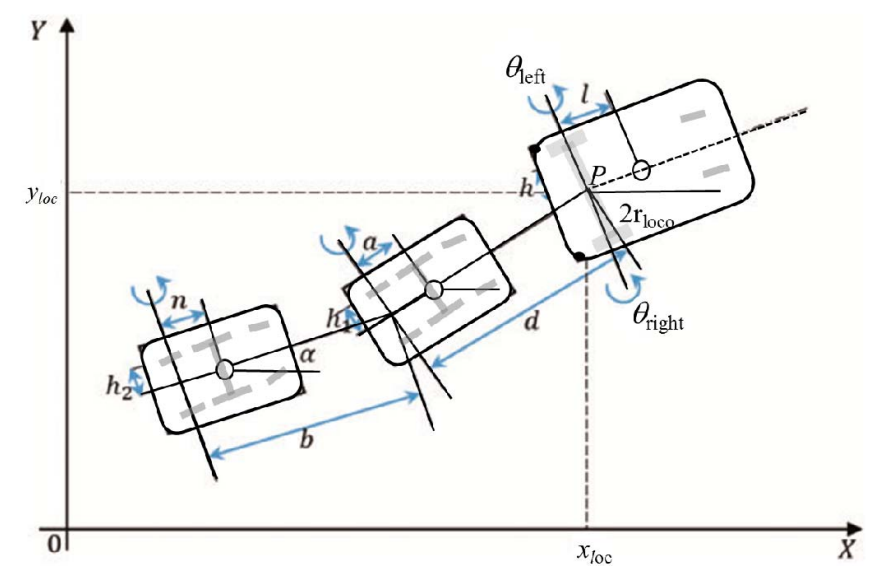

Fig. 17. The vehicle and two carriages consisting of the locomotive and two carriages.

\section{Discussion AND CONCLUSIONS}

The design, construction and control of a fun train consisting of a locomotive and two carriages has been described. Three nonholonomic constraints were assumed. Kinematic and dynamic equations were calculated and a controller proposed to use PD-action filtered errors to track control inputs. The control algorithm performs satisfactorily

The train drivers could take preset responsibility for transporting their child passengers and that provided them with a sense of obligation and authority. Children could feel as if they were a 'train driver' in the locomotive and could safely drive other children around as passengers. The fun train has been used by many children with different special needs. It can accommodate most wheelchair seating systems using three seat adaptors. The wheelbase dimensions have handled multiple loads with various numbers and sizes of passengers in the carriages on slopes and in narrow corridors.

The locomotive looks realistic enough for the children and testing with the Langner Scanning Collision Avoidance Device at the front of the locomotive has shown that local obstacles can be detected and can provide a dead-man switch to stop the train. The train has offered new opportunities for children with complex needs and the locomotive has been successfully driven by a child driver with only a single switch to start and stop the train. All the children who have seen or heard the train have been interested. The ability to accommodate different seating arrangements has allowed friends and children from families to operate and ride in the train. The adaptability of the train seating has allowed children with varying abilities to be passengers. Some children have wanted to drive the train and others have just wanted to be a passenger. When the train was full then other children would follow the train in their powered wheelchairs and staff would push other children behind the train while they were sitting in their wheelchairs or buggies. The train has proved to be an extended group activity. Some children grasped what was going on, especially the children who could already drive a powered wheelchair. Children who were not disabled could still ride in the train with young disabled or non-disabled passengers.

Children on the train have successfully used control devices and infra-red transmitters to interact with toys and responsive systems in play areas and adventurous tunnels.
The fun train is already providing early mobility experiences for children and introducing powered mobility as a fun activity. It is providing opportunities for able bodied and disabled young people to participate in a joint activity together that involves taking turns and sharing.

Simulation and experimental results are presented to show the effectiveness of the controller for the train. Results show that tracking performance is satisfactory despite no a priori knowledge of plant dynamics.

Lyapunov method and Barbalat lemma were used to investigate closed-loop asymptotic stability and that analysis can be obtained from the author on request.

\section{APPENDIX \\ DYNAMIC MODELLING}

If the wheels are assumed to roll without slipping on the ground, then there are nonholonomic constraints in the kinematic model of the vehicle and two carriages. The train is shown in Fig. 1, the kinematic model of the train is shown in Fig. 17 and Fig. 6 considered planar motion for the model.

There are $3 \times$ nonholonomic constraints because of the nonslip conditions of the wheels on the locomotive, the middle carriage and the rear carriage.

That ideal constraint is never completely satisfied, but it is assumed here. The equations in (12) represent constraints between generalized coordinates and velocities:

$$
\begin{aligned}
-(\mathrm{dx} / \mathrm{dt}) \sin \phi+(\mathrm{dy} / \mathrm{dt}) \cos \phi & =0 \\
-(\mathrm{dx} / \mathrm{dt}) \sin \theta+(\mathrm{dy} / \mathrm{dt}) \cos \theta-d(\mathrm{~d} \theta / \mathrm{dt}) & =0 \\
-(\mathrm{dx} / \mathrm{dt}) \sin \alpha+(\mathrm{dy} / \mathrm{dt}) \cos \alpha-d(\mathrm{~d} \theta / \mathrm{dt}) & \\
\cos (\theta-\alpha)-b(\mathrm{~d} \phi / \mathrm{dt}) & =0
\end{aligned}
$$

So that the system constraints in Pfaffian form [50] are:

$\boldsymbol{A}(\boldsymbol{q}) \mathrm{d} \boldsymbol{q} / \mathrm{dt}=0$

$$
A(q)=\left[\begin{array}{ccccc}
-\sin \phi & \cos \phi & 0 & 0 & 0 \\
-\sin \theta & \cos \theta & 0 & -\mathrm{d} & 0 \\
-\sin \alpha & \cos \alpha & -b & -\mathrm{d} \cos (\theta-\alpha) & 0
\end{array}\right]
$$

Let $\boldsymbol{S}(\boldsymbol{q})$ be a matrix such that $\boldsymbol{A}(\boldsymbol{q}) \boldsymbol{S}(\boldsymbol{q})=0$, where $\boldsymbol{S}(\boldsymbol{q})$ is the Jacobian matrix.

A velocity vector $v$ can then be found so that the kinematic model of the vehicle and two carriages (locomotive with two wheeled carriages) is

$$
\mathrm{d} \boldsymbol{q} / \mathrm{dt}=\boldsymbol{S}(\boldsymbol{q}) \boldsymbol{v}
$$

where $\boldsymbol{v}=\left[\begin{array}{ll}v_{\text {lin }} & v_{\text {rot }}\end{array}\right]^{\mathrm{T}}$ and $v_{\text {lin }}$ is the linear velocity at the center of the back wheels of the locomotive, $P$, and $v_{\text {rot }}$ is the rotational velocity of the locomotive as:

$$
\begin{aligned}
& \left.v_{\text {lin }}=V_{P}=+/-\sqrt{ }\{(\mathrm{dx}) / \mathrm{dt})^{2}+(\mathrm{dy} / \mathrm{dt})^{2}\right\} \\
& v_{\text {rot }}=\phi
\end{aligned}
$$

The system Jacobian matrix is $\boldsymbol{S}(\boldsymbol{q})$.

$$
\boldsymbol{S}(\boldsymbol{q})=\left[\begin{array}{cc}
\cos \phi & 0 \\
\sin \phi & 0 \\
0 & 1 \\
(1 / d) \sin (\phi-\theta) & 0 \\
(1 / b) \sin (\theta-\alpha) \cos (\phi-\theta) & 0
\end{array}\right]
$$




$$
\begin{gathered}
{\left[\begin{array}{ccccc}
m & 0 & -m_{3} l \sin \phi & H_{1} \sin \theta & H_{2} \sin \alpha \\
0 & m & m_{3} l \cos \phi & -H_{1} \cos \theta & -H_{2} \cos \alpha \\
-m_{3} / \sin \phi & m_{3} / \cos \phi & \mathrm{I}_{\phi} & 0 & 0 \\
H_{1} \sin \theta & -H_{1} \cos \theta & 0 & \mathrm{I}_{\theta} & H_{2} \mathrm{~d} \cos (\theta-\alpha) \\
H_{2} \sin \alpha & -H_{2} \cos \alpha & 0 & H_{2} \mathrm{~d} \cos (\theta-\alpha) & \mathrm{I}_{\alpha}
\end{array}\right]} \\
{\left[\begin{array}{ccccc}
0 & 0 & -m_{3} /(\mathrm{d} \phi / \mathrm{dt}) \cos \phi & H_{1}(\mathrm{~d} \theta / \mathrm{dt}) \cos \theta & H_{2}(\mathrm{~d} \alpha / \mathrm{dt}) \cos \alpha \\
0 & 0 & -m_{3} /(\mathrm{d} \phi / \mathrm{dt}) \sin \phi & H_{1}(\mathrm{~d} \theta / \mathrm{dt}) \sin \theta & H_{2}(\mathrm{~d} \alpha / \mathrm{dt}) \sin \alpha \\
0 & 0 & 0 & 0 & 0 \\
0 & 0 & 0 & 0 & H_{2}(\mathrm{~d} \alpha / \mathrm{dt}) \sin (\theta-\alpha) \\
0 & 0 & 0 & -H_{2}(\mathrm{~d} \theta / \mathrm{dt}) \sin (\theta-\alpha) & 0
\end{array}\right]} \\
\boldsymbol{B}(\boldsymbol{q})=\frac{1}{\mathrm{r}_{\text {loco }}}\left[\begin{array}{cc}
\cos \phi & \cos \phi \\
\sin \phi & \sin \phi \\
h & -h \\
0 & 0 \\
0 & 0
\end{array}\right] \\
\tau=\left[\begin{array}{c}
\tau_{\text {right }} \\
\tau_{\text {left }}
\end{array}\right]
\end{gathered}
$$

Considering Fig. 7., with $\boldsymbol{q}$, a generalized coordinates vector, then the kinetic system model can be written as:

\begin{tabular}{|c|c|}
\hline$M^{\prime}(q)$ & $\begin{array}{l}\text { is a symmetric, positive definite matrix } \\
\text { [ } 5 \text { by } 5] \text {, }\end{array}$ \\
\hline$C(\boldsymbol{q}, \mathrm{d} \boldsymbol{q} / \mathrm{dt})$ & is a centripetal Coriolis matrix [ 5 by 5 ], \\
\hline$B(q)$ & is an input transformation matrix [ 5 by 2 ], \\
\hline$\tau$ & $\begin{array}{l}\text { is a vector of torques applied to left and } \\
\text { right locomotive driving wheels } 2 \text { by } 1]\end{array}$ \\
\hline $\begin{array}{l}A^{T}(q) \\
\lambda\end{array}$ & $\begin{array}{l}\text { is a system constraint matrix [ } 3 \text { by } 5] \text { and } \\
\text { is a vector of Lagrange multipliers }[3 \text { by } 1] \text {. }\end{array}$ \\
\hline
\end{tabular}

$$
\boldsymbol{M}^{\prime}(\boldsymbol{q}) \mathrm{d}^{2} \boldsymbol{q} / \mathrm{dt}^{2}+\boldsymbol{C}(\boldsymbol{q}, \mathrm{d} \boldsymbol{q} / \mathrm{dt}) \mathrm{d} \boldsymbol{q} / \mathrm{dt}=\boldsymbol{B}(\boldsymbol{q}) \tau+\boldsymbol{A}^{T}(\boldsymbol{q}) \lambda
$$

Where:

Considering these matrices:

$\boldsymbol{M}(\boldsymbol{q})$ is given as (20), as shown at the top of this page, where

$$
\begin{aligned}
m & =m_{1}+m_{2}+m_{3} . \\
\mathrm{I}_{\phi} & =J_{3}+m_{3} l^{2} . \\
H_{1} & =m_{1} \mathrm{~d}+m_{2}(\mathrm{~d}-\mathrm{a}) . \\
\mathrm{I}_{\theta} & =J_{2}+m_{1} \mathrm{~d}^{2}+\mathrm{m}_{2}(\mathrm{~d}-\mathrm{a})^{2} . \\
H_{2} & =m_{1}(b-n) . \\
\mathrm{I}_{\alpha} & =\mathrm{J}_{1}+m_{1}(\mathrm{~b}-\mathrm{n})^{2} .
\end{aligned}
$$

$\boldsymbol{C}(\boldsymbol{q}, \mathrm{d} \boldsymbol{q} / \mathrm{dt})$ is given as (21), as shown at the top of this page, $\mathrm{B}(\mathrm{q})$ is given as (22), as shown at the top of this page, and $\tau$ is given as (23), as shown at the top of this page.

Substituting into equation (8) from equation (4) removes Lagrange multipliers, and then using $\boldsymbol{A}(\boldsymbol{q}) \quad \boldsymbol{S}(\boldsymbol{q})=0$, then:

$$
\boldsymbol{M}^{\prime}(\boldsymbol{q}) d^{2} \boldsymbol{v} / d t^{2}+\boldsymbol{C}(\boldsymbol{q}, \boldsymbol{v}) \boldsymbol{v}=\boldsymbol{B}(\boldsymbol{q}) \tau
$$

Where

$$
\begin{aligned}
\boldsymbol{M}^{\prime}(\boldsymbol{q}) & =\boldsymbol{S}^{\mathrm{T}}(\boldsymbol{q}) \boldsymbol{M}(\boldsymbol{q}) \boldsymbol{S}(\boldsymbol{q}) \in \boldsymbol{R}^{2 \times 2} \\
\boldsymbol{C}^{\prime}(\boldsymbol{q}, \boldsymbol{v}) \boldsymbol{v} & =\boldsymbol{S}^{\mathrm{T}}(\boldsymbol{q}) \boldsymbol{B}(\boldsymbol{q}) \in \boldsymbol{R}^{2 \times 2} \\
\boldsymbol{B}^{\prime}(\boldsymbol{q}) & =\boldsymbol{S}^{\mathrm{T}}(\boldsymbol{q}) \boldsymbol{M}(\boldsymbol{q}) d \boldsymbol{S} / d t(\boldsymbol{q}) \boldsymbol{C}^{\prime}(\boldsymbol{q}, \boldsymbol{v}) \in \boldsymbol{R}^{2 \times 2}
\end{aligned}
$$

The system kinematic and kinetic models are separated so that the kinetic equations have the Lagrange multipliers removed.

\section{REFERENCES}

[1] M. Langner, "Effort reduction and collision avoidance for poweredwheelchairs," Ph.D. dissertation, School Eng., Univ. Portsmouth, Portsmouth, U.K., 2012.

[2] A. M. Cook and J. J. Polgar, Cook and Hussey's Assistive Technologies, 3rd ed. Louis, MO, USA: Mosby, 2007.

[3] T. Felzer, B. Strah, R. Nordmann, and S. Miglietta, "Alternative wheelchair control involving intentional muscle contractions," Int. J. Artif. Intell, vol. 18, no. 3, pp. 439-465, 2009.

[4] J. Kim et al., "Assessment of the tongue-drive system using a computer, a smartphone, and a powered-wheelchair by people with tetraplegia," IEEE Trans. Neural Syst. Rehabil. Eng., vol. 24, no. 1, pp. 68-78, Jan. 2016.

[5] (Jan. 2016). Christopher and Dana Reeve Foundation. One Degree of Separation: Paralysis and Spinal Cord Injury in the United States. [Online]. Available: http://www.christopherreeve.org

[6] B. Martin and L. McCormack, "Issues surrounding assistive technology use and abandonment in an emerging technological culture," in Assistive Technology on the Threshold of the New Millennium, C. Buhler and H. Knops, Eds., Amsterdam, The Netherlands: IOS Press, 1999, pp. 413-420.

[7] B. Yousefi, X. Huo, and M. Ghovanloo, "Quantitative and comparative assessment of learning in a tongue-operated computer input devicePart II: Navigation tasks," IEEE Trans. Inf. Technol. Biomed., vol. 15, no. 5, pp. 457-747, Sep. 2011.

[8] K. Alipour and S. A. A. Moosavian, "Effect of terrain traction, suspension stiffness and grasp posture on the tip-over stability of wheeled robots with multiple arms," Adv. Robot., vol. 26, nos. 8-9, pp. 817-842. 2012.

[9] G. Campion, G. Bastin, and B. D'Andrea-Novel, "Structural properties and classification on kinematic and dynamic models of wheeled mobile robots," Russian J. Nonlinear Dyn., vol. 7, no. 4, pp. 733-769, 2011.

[10] G. Campion, B. D'Andrea-Novel, and G. Bastin, "Controllability and state feedback stabilizability of non holonomic mechanical systems," in Advanced Robot Control. Heidelberg, Germany: Springer, 1991, pp. $106-124$.

[11] I. Kolmanovsky and N. McClamroch, "Developments in nonholonomic control problems," IEEE Control Syst., vol. 15, no. 6, pp. 20-36, Jun. 1995.

[12] A. De Luca and G. Oriolo, Modelling and Control of Nonholonomic Mechanical Systems. New York, NY, USA: Springer, 1995.

[13] B. D'Andrea-Novel, G. Bastin, and G. Campion, "Modelling and control of non-holonomic wheeled mobile robots," in Proc. IEEE Int. Conf. Robot. Autom., New York, NY, USA, Apr. 1991, pp. 1130-1135. 
[14] T. Das, I. Kar, and S. Chaudhury, "Simple neuron-based adaptive controller for a nonholonomic mobile robot including actuator dynamics," Neurocomputing, vol. 69, no. 16, pp. 2140-2151, 2006.

[15] A. De Luca, G. Oriolo, and C. Samson, "Feedback control of a nonholonomic car-like robot," in Robot Motion Planning and Control, New York, NY, USA: Springer, 1998, pp. 171-253.

[16] H. Chowdhry, Model Predictive Control of Wheeled Mobile Robots, Univ. Ontario Institute of Technology: Oshawa, ON, Canada, 2010.

[17] A. Keymasi K. M. R. Bidgoli and S, Ali A. Moosavian, "Non-modelbased control for a wheeled mobile robot towing two trailers," IMechE Part K, J. Multi-Body Dyn., vol. 229, no. 1, pp. 97-108, 2015.

[18] S. A. A. Moosavian, M. R. Bidgoli, and A. Keymasi, "Tracking control of a wheeled mobile robot with two carriages," presented at the 21 st Annu. Int. Conf. Mech. Eng., Tehran, Iran, 2013.

[19] D. A. Sanders, "Using self-reliance factors to decide how to share control between human powered wheelchair drivers and ultrasonic sensors," IEEE Trans. Neural Syst. Rehabil. Eng., vol. 25, no. 8, pp. 1221-1229, Aug. 2017, doi: 10.1109/TNSRE.2016.2620988.

[20] F. Pourboghrat and M. P. Karlsson, "Adaptive control of dynamic mobile robots with nonholonomic constraints," Comput. Electr. Eng., vol. 28, no. 4, pp. 241-253, 2002.

[21] F. N. Martins et al., "An adaptive dynamic controller for autonomous mobile robot trajectory tracking," Control Eng. Pract., vol. 16, no. 11, pp. 1354-1363, 2008.

[22] B. S. Park et al., "Adaptive neural sliding mode control of nonholonomic wheeled mobile robots with model uncertainty," IEEE Trans. Control Syst. Technol., vol. 17, no. 1, pp. 207-214, Jan. 2009.

[23] E. S. Elyoussef et al., "On a wheeled mobile robot trajectory tracking control: 1st and 2nd order sliding modes applied to a compensated inverse dynamics," in Proc. 11th Pan-Amer. Congr. Appl. Mech., Brazil, South America, 2010, pp. 1-6.
[24] A. K. Khalaji and S. A. A. Moosavian, "Robust adaptive controller for a locomotive-carriage mobile robot," IEEE/ASME Trans. Mechantron., vol. 19, no. 4, pp. 943-953, Apr. 2014.

[25] M. Karimi and S. A. A. Moosavian, "Modified transpose effective Jacobian law for control of underactuated manipulators," Adv. Robot., vol. 24, no. 4, pp. 605-626, 2010.

[26] F. M. Raimondi and M. Melluso, "A new fuzzy robust dynamic controller for autonomous vehicles with nonholonomic constraints," Robot. Auto. Syst., vol. 52, no. 2, pp. 115-131, 2005.

[27] J. Velagic, N. Osmic, and B. Lacevic, "Neural network controller for mobile robot motion control," Int. J. Intell. Syst. Tech., vol. 3, no. 2, pp. 127-133, 2008.

[28] A. K. Khalaji and S. A. A. Moosavian, "Modified transpose Jacobian for control of a locomotive-carriage wheeled robot," presented at the Int. Conf. Robot. Mechatronics, 2013.

[29] D. A. Sanders, A. Gegov, S. Benjamin, N. David, and G. Alexander, "Rule-based system to assist a powered wheelchair driver," in Proc. IEEE Intell. Syst. Conf., 2017.

[30] D. A. Sanders, B. Sanders, A. Gegov, and N. David, "Results from investigating powered wheelchair users learning to drive with varying levels of sensor support," in Proc. IEEE Intell. Syst. Conf., 2017.

[31] D. Sanders and N. Bausch, Improving Steering of a Powered WheelChair Using an Expert System to Interpret Hand Tremor (Lecture Notes in Computer Science), vol. 9245. London, U.K.: Springer, 2015, pp. 460-471, doi: 10.1007/978-3-319-22876-1_39.

[32] D. A. Sanders, I. Stott, J. Graham-Jones, A. Gegov, and G. Tewkesbury, "Expert system to interpret hand tremor and provide joystick position signals for powered wheelchairs with ultrasonic sensor systems," Ind. Robot, Int. J., vol. 38, no. 6, pp. 585-598, 2011. 